\title{
Crise ou transformations du monde du travail dans les Amériques?
}

\section{Donna Kesselman}

\section{(2) OpenEdition}

\section{Journals}

Édition électronique

URL : https://journals.openedition.org/ideas/1022

DOI : 10.4000/ideas. 1022

ISSN : 1950-5701

Traduction(s) :

Transformations in the labor market in the Americas - or is it crisis? - URL : https:// journals.openedition.org/ideas/3073 [en]

¿Crisis o transformación del mundo laboral en las Américas? - URL : https://journals.openedition.org/ ideas/3079 [es]

Crise ou transformação do mundo dos negócios nas Américas? - URL : https://

journals.openedition.org/ideas/4722 [pt]

\section{Éditeur}

Institut des Amériques

\section{Référence électronique}

Donna Kesselman, « Crise ou transformations du monde du travail dans les Amériques ? », IdeAs [En ligne], 5 | 2015, mis en ligne le 18 mai 2015, consulté le 19 octobre 2022. URL : http:// journals.openedition.org/ideas/1022 ; DOI : https://doi.org/10.4000/ideas.1022

Ce document a été généré automatiquement le 19 octobre 2022.

\section{cc)}

Creative Commons - Attribution - Pas d'Utilisation Commerciale - Pas de Modification 4.0 International - CC BY-NC-ND 4.0

https://creativecommons.org/licenses/by-nc-nd/4.0/ 


\title{
Crise ou transformations du monde du travail dans les Amériques?
}

\author{
Donna Kesselman
}

Numéro coordonné par Donna Kesselman

Rédactrice en chef : Isabelle Vagnoux

Comité de rédaction : ont contribué à l'élaboration de ce numéro Christian Azaïs, Virginie Baby Collin, Vincent Dubreuil, Jean Kempf, Alvar de la LIosa, Delphine Mercier, Modesta Suarez Secrétariat de rédaction : Claire Bouffard (secrétaire de rédaction), Anne-Julie Kerebel (stagiaire recherche et publications)

1 Y a-t-il une crise du travail dans les Amériques? Si oui, en quoi est-elle propre à cet hémisphère en particulier? Comment se-décline-t-elle du Nord au Sud et dans chacun des pays? Ou bien cela revient-il à appréhender les configurations émergentes qui caractérisent la globalisation du marché du travail du XXI siècle?

2 Telle est la question que pose ce numéro d'IdeAs, dans la suite du précédent numéro consacré aux "Crises et effets de crise dans les Amériques", traitant ici plus particulièrement de phénomènes liés au monde du travail.

3 Il est devenu coutumier de pointer du doigt la crise du travail qui frappe les pays industrialisés depuis une génération. La globalisation financière et les innovations techniques et technologiques se combinent pour faire tomber les frontières $d u$ commerce comme de l'information, entraînant des politiques publiques néolibérales qui minent les institutions historiques de régulation et de redistribution sociale. La Great Recession de 2008 n'a donc fait, en ce qui concerne le marché du travail et de l'emploi, qu'accélérer des processus déjà en cours. Ce constat, d'ordre général, appelle un certain nombre de précisions.

4 La globalisation, loin d'être homogène, est faite de standardisation et surtout de différenciation (Azaïs C., 2010). Il s'agit d'un processus non uniforme, globalizing world labor market, dont il faut apprécier les particularités nationales, celles d'autres échelles institutionnelles, en même temps que les interactions entre acteurs sociaux multiples (Kesselman D., 2010a ; Giraud O., 2012). En ce qui concerne le travail, la différenciation 
vient, d'une part, de résistances collectives et individuelles aux pressions venant des entreprises transnationales pour toujours plus de flexibilité et de baisse du coût du travail. Elles s'expriment à travers des mouvements sociaux pour la défense du transport public, de la santé et de l'éducation dans un pays aussi dynamique que le Brésil, tout autant que dans la création de droits sociaux dans un pays aussi invraisemblable que les États-Unis, où certains États fédérés imposent désormais aux entreprises - pour la première fois venant de la part de puissances publiques l'attribution aux salariés de congés rémunérés (Thévenard E., 2015). D’autre part, en faisant la part belle aux pressions des intérêts locaux, la flexibilité favorise aussi la différentiation.

5 Certes, du Sud au Nord des Amériques, il existe des réalités comparables sinon communes : dans le numéro précédent d'IdeAs, Laurence Whitehead propose une série de facteurs contextuels plaidant en faveur d'une spécificité d'expériences dans ce qui peut s'appeler le « Nouveau Monde» (Whitehead L., 2013). Les pays d'Amérique latine partagent le fait d'appartenir aux pays dits du «Sud»-Global South -, notion qui s'inscrit dans le développement du capitalisme. L'appellation plus récente que l'ancienne de "pays sous-développés » ou « du tiers monde » se réfère aux économies nationales qui ne bénéficient pas d'accès direct au marché mondial. Anciennement dominés, voire colonisés, par les pays développés, ils ont subi la prospérité marchande puis capitaliste, en raison du déplacement de leurs propres richesses afin de nourrir celles des pays du Nord. Certains sont considérés aujourd'hui, selon le langage politiquement correct, comme des pays en développement, d'autres comme des pays émergents. Bien que le sens de cette dernière catégorie évolue rapidement (Salama P., 2014), le sous-entendu prête à la confusion.

6 Cette dernière appellation, à l'instar de celle datant des années 1960 de "pays en voie de développement", implique l'existence d'une trajectoire historique linéaire qui emboîterait le pas à celle des pays du Nord, passant par leur propre révolution industrielle et post-industrielle. Or, cette conception d'un schéma lisse de la croissance capitaliste des démocraties occidentales traditionnelles ne correspond pas à l'évolution de la division internationale du travail. De surcroît, les environnements sociopolitiques propres à chaque pays du Sud et les tensions qui caractérisent le marché mondial excluent toute vision simplifiée, sinon glorifiée, d'une reproduction de l'histoire économique.

7 La crise de 2008 serait venue confirmer l'hétérogénéité des économies latinoaméricaines. Comme le démontre Carlos Quenan, cette "réalité de trajectoires économiques diversifiées » s'applique même à des pays à plusieurs égards comparables, tels que le Mexique et le Brésil. Plusieurs facteurs se sont conjugués dans ce dernier pays pour atténuer l'impact de la crise, alors qu'au Mexique les conséquences ont été spécialement "virulentes sur l'économie comme sur la population». Les pays dépendant principalement des revenus de l'exportation d'hydrocarbures ont réagi chacun de manière différente face à la crise (Quenan C., 2013).

Ces réactions pourraient s'expliquer par l'existence de modèles nationaux différents. Il existerait même des « variétés du capitalisme » (Hall P. et D. Soskice, 2001) spécifiques à l'Amérique latine, articulées entre deux pôles constitués par les deux pays se prévalant des plus grandes économies (Bizberg I., 2012 ; Théret B., 2002 ; Marques-Pereira J. et B. Théret, 2004), se distinguant tout singulièrement sur le terrain du travail. Selon Bizberg, le Brésil peut être qualifié de «State-led/inward oriented» où l'État joue un 
rôle central dans l'orientation de l'activité économique vers le marché interne, renforçant de cette manière les fondements d'une intégration active dans le marché mondial. L'article dans le présent numéro d'IdeAs proposé par Marcia Leite et Carlos Salas semble conforter cette hypothèse. Cette étude sur l'évolution des métiers fait état d'une réduction des inégalités grâce à l'accès croissant par des populations défavorisées à des postes de travail plus qualifiés, une tendance qui s'affirme malgré la période de la crise et qui s'inscrit dans le modèle brésilien de développement. Dans l'autre pôle et dans l'extrême inverse incarné par le Mexique, l'État n'occupe qu'une place subsidiaire d'articulation de la structure productive qui se tourne avant tout vers l'extérieur. Il restreint son action à la régulation et à la promotion du capital national grâce à l'intervention contre-cyclique, dans ce qu'on peut appeler « State regulated/externallyoriented capitalism ». Cette perception correspond à celle d'Élodie Segal, comme on le lira plus bas, qui qualifie la société mexicaine comme étant «défigurée par des inégalités». L'analyse fait part d'un modèle de production qui, au nom de la modernisation du pays, s'oriente selon les besoins de la finance et des clientsmandateurs. Les pressions internes qui en découlent conduisent à la dérégulation, à l'individualisation et - pour la population la plus fragile - à la réduction des droits sociaux des travailleurs. C'est en effet le cas du Mexique, où le salaire minimum est parmi ceux les plus bas d'Amérique latine.

9 D'autres pays, comme le Chili, réunissent des traits caractéristiques des deux modèles (Bizberg I., 2012). Et c'est aussi en ces termes qu'ici, Guillermo Wormald et Maria Paz Trebilcock qualifient ce pays. Leur analyse du rapport entre travail et cohésion sociale en milieu urbain au Chili met en évidence l'émergence d'une "nouvelle société marchande " qui viendrait bouleverser les rapports anciens, en s'imposant toujours plus comme un mécanisme de coordination des opportunités d'accès au travail. Dans quelle mesure le travail sert-il toujours de vecteur de création d'un espace de rencontre, de valorisation sociale et de citoyenneté ?Alors que vers la fin du siècle dernier l'Amérique latine avait été présentée comme un possible "laboratoire social » de l'avenir du travail des pays du Nord, position reprise plus tard par Beck à travers l'idée de «brésilianisation de l'Occident » (Beck Ü., 2000), cette expérience chilienne vérifie, à nos yeux, notre hypothèse développée dans ce numéro, et par d'autres, selon laquelle des pratiques en cours aux États-Unis, quant à l'articulation du travail et des prestations sociales à partir du marché (market based), pourraient inverser le sens de l'expérimentation (Kesselman D., 2010b).

10 L'affirmation d'une globalisation axée sur la différenciation, entre et à l'intérieur des économies et des sociétés, se justifie ainsi particulièrement en ce qui concerne les marchés du travail et de l'emploi. Les situations nationales ne sont que difficilement comparables en raison des spécificités historiques dans la construction de chaque État social et d'un système de relations professionnelles. À titre d'exemple, le caractère minimaliste et fragmenté des institutions de réglementation sociale aux États-Unis aboutit à de grands écarts, d'une région à l'autre, d'une entreprise à l'autre, dans les caractéristiques de la relation de l'emploi (employment relationship). L'ensemble s'éloigne considérablement du système de son voisin canadien, bien plus protecteur (Lapointe P.-A., 2013 ; Kesselman D., 2007). Pour les pays du Sud, il faut prendre en compte à la fois l'histoire sociopolitique et les différences dans le degré et les stratégies de développement. 
11 Certaines contributions de ce numéro s'intéressent aux relations professionnelles et aux luttes collectives qui visent, dans leur contexte national, pallier aux conséquences de la mondialisation néolibérale. Malgré son affaiblissement, le contre-pouvoir du syndicalisme n'a pas dit son dernier mot.

12 Les usines de confection représentent un des principaux secteurs ayant enregistré une augmentation du travail formel au Guatemala, et notamment pour les femmes, dans ce pays où la vaste majorité de la population active fait l'objet d'activités informelles. Mais ces maquilas font preuve des pires conditions de travail et de précarité, tout en appliquant des pratiques antisyndicales féroces. Les travailleuses ne baissent pas pour autant les bras devant des obstacles et contraintes quotidiennes dressés contre l'exercice des droits syndicaux, comme nous le révèle Quentin Delpech, à partir d'enquêtes de terrain.

13 Le Venezuela est un cas exceptionnel. Thomas Posado nous propose un regard de l'intérieur du mouvement syndical des bouleversements qui ont lieu depuis l'avènement du régime chaviste. Ce pouvoir, qui se réclame des intérêts populaires et d'une politique de résistance aux injonctions des puissances économiques et financières mondiales, a influé sur le positionnement des organisations ouvrières forgées dans un temps révolu et sur le renouvellement du syndicalisme dans ce pays.

14 IdeAs présente également dans ce numéro une analyse comparative du syndicalisme nord-américain. Comment expliquer l'existence aux États-Unis d'un taux de syndicalisation parmi les plus bas du monde occidental et alors qu'au Canada, il est parmi les plus élevés? Dans une interview Paul-André Lapointe, spécialiste des relations professionnelles à l'Université Laval à Québec, explique cette situation apparemment paradoxale étant donné les similitudes socioéconomiques et l'histoire commune qui ont rapproché le mouvement syndical des deux pays : l'instauration de la même législation de gestion des relations professionnelles dans l'industrie et la constitution de ce qui s'appelle les International Unions, à savoir des organisations syndicales implantées à la fois aux États-Unis et au Canada. Le récit trace cette évolution croisée, puis de plus en plus divergente à partir des années 1960, ainsi que les défis auxquels sont confrontés aujourd'hui les travailleurs et leurs organisations. Le prisme des droits syndicaux offre aussi un aperçu de la culture politique et sociale très variée entre les diverses provinces canadiennes.

15 Ces expériences nationales de croissance économique et de défense des intérêts sociaux collectifs donnent lieu à des différenciations en termes d'égalité sociale. Toute appréciation de la situation au et du travail, du Sud au Nord et jusqu'au sein des sociétés particulières, doit dépasser les statistiques macroéconomiques. Des taux de croissance élevés et l'extension du salariat à travers l'Amérique latine, malgré la crise, font rêver ses voisins du Nord. Ils n'ont pas pour autant abouti à la construction d'une relation d'emploi salarial accompagnée de prestations sociales au niveau atteint historiquement par celles au Nord; on atteste, dans cette région aussi, de la multiplication d'emplois précaires (Giglia A., 2014 ; Rosenfield C., 2010).

De même, l'informalité n'a pas pour autant diminué dans le sous-continent, elle a tout au plus changé de visage et de nature (Azaïs C. et M. Lehalleur, 2014). Guillermo Wormald et Maria Paz Trebilcock illustrent la complexité du phénomène au Chili : l'emploi formel sous forme de salarisation croît pour les hommes comme pour les femmes, de tous les âges et dans l'ensemble des déciles de revenus et de surcroît sous forme de CDI (contrat à durée indéterminée). Il en demeure que ces contrats, sauf pour 
le dernier décile (les $10 \%$ de salariés les mieux rémunérés), sont loin d'être aussi protecteurs que ceux qu'ont connus, notamment, les salariés européens.

En conséquence, à l'heure de la mondialisation néolibérale, la question se pose de savoir quelles perspectives sociales existent, même dans des économies promises à un développement sans précédent, si le cadre de redistribution sociale le plus étendu des pays du Nord est voué inexorablement à se rétrécir.

L'histoire n'est pourtant pas, bien sûr, écrite d'avance. Les apports de ce numéro d'IdeAs nous aident à reposer, dans des termes des plus actuels, la question du départ : existe-il une crise du travail ou bien se trouve-t-on en présence de configurations nouvelles, des configurations caractéristiques de la globalisation qui augurent le siècle nouveau (D’Amours M. et al., 2015) ? La réponse dépendra non seulement du jeu des acteurs mais également de la santé du système.

Une nouvelle phase de reprise avait été annoncée suite à la crise planétaire déclenchée par l'effondrement boursier étasunien de 2008. Au début de 2014, le quotidien français Le Monde fanfaronne: "Le FMI entrevoit une reprise 'plus forte et plus large'" (Guélaud C., 2014). Par la suite, le FMI fait marche arrière : à la veille du $45^{\mathrm{e}}$ Forum économique mondial de Davos, en janvier 2015, le FMI revoit ses prévisions à la baisse. L'affaiblissement de l'investissement et de la croissance potentielle dans la plupart des "grands pays " s'affirme, la reprise dans la zone euro et au Japon sera plus lente que prévue. Quant aux pays émergents et en développement, y compris les BRICS dont le dynamisme a été tant célébré au début des années 2000, ils traversent une période difficile, notamment l'Afrique subsaharienne et l'Amérique latine. Celle-ci est due entre autres à la baisse des cours des produits de base. Seule l'économie des États-Unis connaît des projections revues à la hausse (FMI, 2015).

20 Volant d'entraînement de l'économie mondiale, l'économie étasunienne qui se dynamise devrait s'avérer porteuse pour le marché du travail, en commençant par celui de son propre pays. Pourtant, ce n'est pas ce qu'écrit Donna Kesselman dans son article: selon elle, le modèle d'emploi, l'American Job Machine, connaît une crise structurelle. Olivier Frayssé pose les enjeux dans la longue durée : la quasi-disparition de la retraite par répartition aux États-Unis a des implications qui vont bien au-delà du sort de cette prestation sociale, car elles sont révélatrices de l'éclipse des fondements mêmes du rêve américain. Enfin, Christian Azaïs propose une réflexion théorique sur les transformations du travail dans un globalizing world: s'agit-il d'une amplification du processus déjà en cours ou bien d'une crise accompagnée d'un changement de nature ?

21 Aux lectrices et aux lecteurs d'IdeAs d'en juger. Ce panorama de recherches d'une si grande richesse, livré par seize auteurs travaillant sur sept pays des Amériques, offre des perspectives d'analyse multiples - sur l'évolution et l'émergence de marchés du travail et de l'emploi, sur le caractère mouvant des phénomènes, sur le rôle des acteurs anciens et nouveaux qui sont autant d'indicateurs du devenir des sociétés et qui nourriront, nous l'espérons, leurs réflexions. 


\section{BIBLIOGRAPHIE}

Azaïs, Christian, "Amérique du Sud : Travail ; Syndicalisme », in Michel Bertrand, Antoine Coppolani, Isabelle Vagnoux, Jean-Michel Blanquer (dir.), Dictionnaire des Amériques, Paris, Laffont Collection Bouquins, à paraître en 2016.

Azaïs, Christian (dir), «Introduction », Labour and Employment in a Globalizing World : Autonomy, collectives and political dilemmas, Brussels, Peter Lang. 2010.

Azaïs Christian et Pepin-Lehalleur Marielle (dir.), Modes de gouvernance dans quatre métropoles latino-américaines (Buenos Aires, Caracas, Mexico et São Paulo) : entre logiques institutionnelles et acteurs, Bruxelles, P.I.E. Peter Lang Ed., 2014.

Beck, Ülrich, The Brave New World of Work, Oxford, Polity Press, 2000.

Bizberg, Ilan, « Types of capitalism in Latin America », Revue Interventions économiques, $\mathrm{n}^{\circ} 47,2012$, p. 1-26, http://interventionseconomiques.revues.org/1772.

D’Amours, Martine, Soussi, Sid Ahmed, Tremblay, Diane-Gabrielle (dir.), Repenser le travail : Des concepts nouveaux pour des réalités transformées, Québec, Presses de l'Université de Québec, 2015.

FMI, Fonds monétaire international, « Les prévisions de croissance mondiale sont révisées en baisse malgré le repli des prix du pétrole et l'accélération de la croissance américaine », Bulletin du FMI, 20 janvier 2015, http://www.imf.org/external/French/pubs/ft/survey/so/2015/ NEW012015AF.htm, consulté le 2 avril 2015.

Giglia, Angela, « Trabajo precario y redes de solidaridad. El caso de los gasolineros en la ciudad de México ", in Angela Giglia et Adelina Miranda (coords), Precariedad urbana y lazos sociales. Una perspectiva comparativa entre México e Italia, México, UAM-Juan Pablos editores, p. 109-137, 2014.

Giraud, Olivier, « L'analyse scalaire des régimes d'action publique en Europe : l'apport méthodologique des comparaisons internationales ", Revue internationale de politique comparée, vol. $19, \mathrm{n}^{\circ} 2$, p. 15-36, 2012.

Guélaud, Claire, "Le FMI entrevoit une reprise 'plus forte et plus large' », Le Monde, 8 avril 2014, http://www.lemonde.fr/economie/article/2014/04/08/le-fmi-entrevoit-une-reprise-plus-forteet-plus-large_4397717_3234.html\#ZAXV65cyXjha3dYj.99, consulté le 5 mars 2015.

Hall, Per A., Soskice, David, Varieties of Capitalism, Oxford University Press, 2001.

Kesselman, Donna, « Travail et salariat aux États-Unis : Quels droits, quelles perspectives?», Revue Française d'Etudes Américaines, n 111, 2007, p. 6-26.

Kesselman, Donna, « A Globalizing labour and employment market » (Postface), in Christian Azaïs (Dir.), Labour and Employment in a Globalizing World: Autonomy, collectives and political dilemmas, Brussels, Peter Lang. 2010a, p. 267-273.

Kesselman, Donna, « Trabalho precário e precarização institucional nos Estados-Unidos », (« Travail précaire et précarisation institutionnelle aux États-Unis »), Sociologias, vol. 12, $n^{\circ} 25$, novembre/décembre, 2010b, p. 66-100.

Lapointe, Paul-André (Dir.), La qualité du travail et de l'emploi au Québec. Données empiriques et cadres conceptuels, Québec, Presses de l'Université Laval, 2013. 
Marques Pereira, Jaime et Théret, Bruno, « Mediaciones institucionales de regulación social y dinámicas macroeconómicas : los casos de Brasil y México » in Carlos Alba et Ilán Bizberg (Dir.), Democracia y Globalización en México y Brasil, México, El Colegio de México, 2004.

Quenan, Carlos, « América latina frente a la crisis económica internacional : buena resistencia global y diversidad de situaciones nacionales ", IdeAs, $\mathrm{n}^{\circ}$ 4, Automne 2013, http:// ideas.revues.org/780.

Rosenfield, Cinara (dir), «Trabalho, emprego e pracarização social », Sociologias, vol n $12, n^{\circ} 25$, novembre/décembre 2010 .

Salama, Pierre, Des pays toujours émergents ?, Paris, La Documentation Française, 2014.

Théret, Bruno, Protection Sociale et Fédéralisme. L'Europe dans le miroir de l'Amérique du Nord, Montréal, Presse de l'Université de Montréal - Peter Lang, 2002.

Thevenard, Evelyn, « Paid Family Leave as Public Policy : A View from the States », in Gérard Gomez et Donna Kesselman (Dir.), Les femmes au travail dans les Amériques, Aix-en-Provence, Presses Universitaires de Provence (PUP), à paraître en 2015.

Whitehead, Laurence, “'Crisis' in the Americas : Is there a Regionally Distinctive Kind ? ", IdeAs, $\mathrm{n}^{\circ}$ 4, Automne 2013, http://ideas.revues.org/611.

\section{AUTEUR}

\section{DONNA KESSELMAN}

Donna Kesselman est Professeure des Universités à l'Université Paris-Est Créteil. Elle publie largement sur les questions du travail, de l'emploi et des relations professionnelles, en proposant une perspective comparative, (France, États-Unis, Brésil, Canada). Elle est co-rédactrice d'un recueil d'articles publié aux Presses Universitaires de Provence, articles issus des communications qui ont été faites lors du Congrès de l'IDA de 2013 "Les Femmes dans les Amériques", et plus particulièrement de l'axe thématique "Les femmes et le travail dans les Amériques". Elle a co-rédigé des études de la DARES sur les relations professionnelles. Elle est au bureau du RT 18 de l'Association Française de Sociologie traitant des relations professionnelles. 